\title{
SPECIAL SECTION: ARTICLE \\ Fundamental Issues in Game Theory and Market Design \\ Introduction to fundamental issues in game theory and market design
}

\author{
Michihiro Kandori ${ }^{1} \cdot$ Takashi Ui $^{2}$
}

Published online: 27 November 2019

(c) Japanese Economic Association 2020

\begin{abstract}
We introduce this special issue, which collects recent developments in game theory and market design. The first half of the special issue consists of five papers on incomplete information games, most of which contribute to the study of robustness of equilibria to incomplete information. The second half of the special issue is devoted to two papers on matching and market design, which provide extension of matching in some important directions. This introduction gives a summary of the special issue by briefly discussing each paper.
\end{abstract}

Since this journal published the last special issue on game theory in 2005 (cf. Imai and Okada 2005), game theory has become much more relevant as an essential tool in economic analysis, which is evident in the list of Nobel Memorial Prize laureates in Economics who study or apply game theory. In particular, the past decade has seen substantial development in market design and mechanism design as applications of game theory.

This special issue is devoted to papers on game theory and market design, particularly incomplete information games (Harsanyi 1967-1968) and matching (Gale and Shapley 1962). In both models, players or individuals are assumed to have their own information or preferences unknown to others, but the goals of analysis can differ. In incomplete information games, one of the goals is to analyze the effects of higher order beliefs on outcomes; in matching, it is to analyze the conflict between stability and other constraints. We collect recent developments in these issues.

The first half of the special issue consists of five papers on incomplete information games. In particular, the first four papers build on the work of Kajii and Morris (1997) who introduce the notion of robustness of equilibria to incomplete information. Given a complete information game, they consider a nearby

Takashi Ui

oui@econ.hit-u.ac.jp

1 The University of Tokyo, Tokyo, Japan

2 Hitotsubashi University, Tokyo, Japan 
incomplete information game with the same players and the same action space, and call it an elaboration of the complete information game. Robustness is defined in terms of elaborations: an equilibrium of a complete information game is robust to all elaborations if every elaboration has a Bayesian Nash equilibrium in a neighborhood of the equilibrium. For example, Kajii and Morris (1997) show that a $\boldsymbol{p}$-dominant equilibrium with $\Sigma p_{i}<1$ is robust to all elaborations (see Fig. 1; Table 1).

The first paper by Kajii and Morris provides a unified framework to survey and relate the notion of robustness and the classic refinement literature by proposing alternative notions of robustness. A canonical elaboration is a special case of an elaboration where each type has either the same payoff function as that in the complete information game or a strictly dominant action. Fixing a subclass of canonical elaborations satisfying some property $X$, the authors ask whether an equilibrium is robust to canonical elaborations satisfying $X$, i.e., whether every canonical elaboration satisfying $X$ has a Bayesian Nash equilibrium in a neighborhood of the equilibrium. The authors also ask whether an equilibrium is justified by some canonical elaborations satisfying $X$, i.e., whether some canonical elaboration satisfying $X$ has a Bayesian Nash equilibrium in a neighborhood of the equilibrium. Then, by choosing an appropriate property $X$, the paper demonstrates that each criterion is equivalent to some classic refinement criterion. For example, a canonical elaboration is said to be independent if types with strictly dominant actions are independently distributed, and robustness to independent canonical elaborations is equivalent to strict perfection in the sense of Okada (1981) (see Fig. 1; Table 1). Since this paper was written in 1997 as a discussion paper, it has been an influential paper supplementing Kajii and Morris (1997) and promoting their idea. Following the authors' decision, we publish it as it was

Fig. 1 The relationship between different types of elaborations

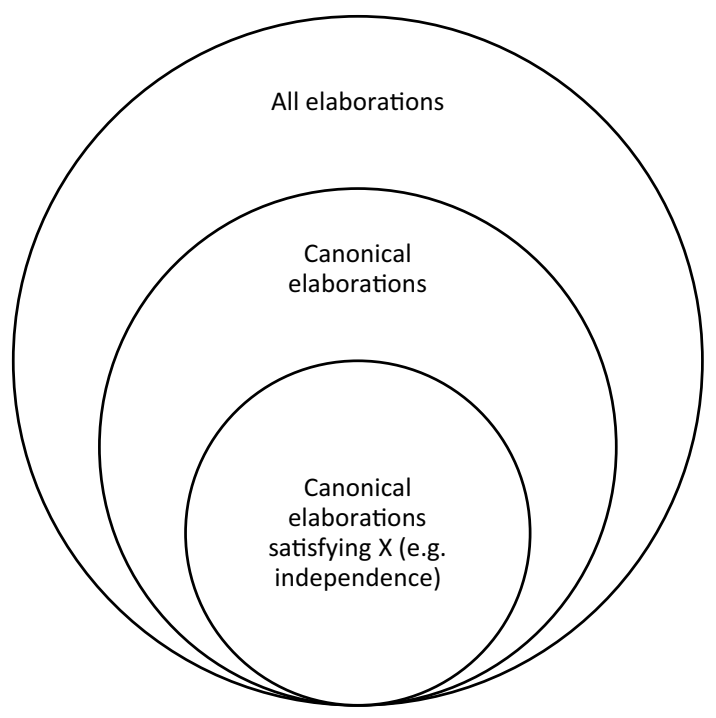


Table 1 Different types of elaborations and robustness

\begin{tabular}{llcc}
\hline $\begin{array}{l}\text { Types of elabora- } \\
\text { tions }\end{array}$ & All elaborations & Canonical elaborations & $\begin{array}{c}\text { Independent canonical } \\
\text { elaborations }\end{array}$ \\
\hline $\begin{array}{l}\text { Examples of } \\
\text { robust equilibria }\end{array}$ & $\begin{array}{c}\text { p-dominant equilibria (Kajii } \\
\text { and Morris 1997) }\end{array}$ & $\begin{array}{c}\text { Potential maximizing } \\
\text { equilibria (Ui 2001) }\end{array}$ & $\begin{array}{c}\text { Strict perfect equilibria } \\
\text { (Okada 1981) }\end{array}$ \\
\hline
\end{tabular}

originally written (including their affiliations) together with a supplement, which is the next paper.

The second paper by the same authors updates a survey part on the notion of robustness in the first paper. It provides notes on relevant developments in the literature, including the next two papers, and how they relate to the first paper.

The third paper by Takahashi solves an open problem which has been known since the first paper was written in 1997. By definition, if an equilibrium is robust to all elaborations, it is also robust to canonical elaborations. Canonical elaborations have played an important role in the literature, because all of the known examples of non-robustness to all elaborations are those of non-robustness to canonical elaborations, such as an email game of Rubinstein (1989). In addition, Ui (2001) shows that if a game has a potential function, then a potential maximizer is robust to canonical elaborations, leaving open whether it is also robust to all elaborations (see Fig. 1; Table 1). This paper demonstrates that robustness to canonical elaborations is strictly weaker than robustness to all elaborations by constructing an example, where the set of potential maximizers is robust to canonical elaborations, but not to all elaborations.

The fourth paper by Murayama studies robustness of solutions in finite depth of reasoning models such as the level-k and cognitive hierarchy models (Stahl and Wilson 1994; Nagel 1995; Camerer et al. 2004), where each player is endowed with an anchor behavior and a set of reasoning levels. The paper provides a general framework to analyze the robustness of predictions to small uncertainty about an anchor behavior. The main result gives a sufficient condition for the robustness: a $\boldsymbol{p}$-dominant equilibrium is robust if players put sufficiently small probability, which is decreasing in $\boldsymbol{p}$, on other players having high reasoning levels. Its corollary reveals the following distinction between the level-k model and the cognitive hierarchy model. A $\boldsymbol{p}$-dominant equilibrium in the level-k model is robust if $p_{i}<1 / I$ for each $i$, where $I$ is the number of players. On the other hand, a $\boldsymbol{p}$-dominant equilibrium in the cognitive hierarchy model is robust if $p_{i}<1$ for each $i$.

The fifth paper by Ui incorporates a general information structure into the Lucas imperfect information model (Lucas 1972) and studies its implications. In the original Lucas model, where information except an unaggregated price is common knowledge, output responds to unanticipated monetary shocks. Contrastingly, in the Lucas model with imperfect common knowledge studied in this paper, output responds to anticipated monetary shocks as well. The author considers an information structure composed of public and private signals and finds the following. When the precision of private information is fixed, the real effects are small not only when a public signal is very precise but also when it is very imprecise. This implies that 
a more precise public signal can amplify the real effects and make the economy more volatile. It is also shown that an outcome in the Lucas model is mathematically equivalent to a Bayesian Nash equilibrium in a team (Radner 1962), which is a special case of a Bayesian potential game with an identical payoff function.

The second half of the special issue is devoted to two papers on matching and market design. Matching theory has been successful in designing rules that can be used in real-life problems to match agents to agents or agents to institutions. Examples include matching medical doctors to hospitals in the residency programs and matching students to public schools in the school choice programs. A prominent feature of those matching problems is that it does not entail any monetary payments, and therefore, there are no "prices" to guide the participants' behavior. Matching theory artificially designs "markets" or sets of rules to determine who is matched to whom, which function well in the absence of the price mechanism. After the initial success in designing the matching rules for the medical doctors and hospitals in the US and other countries, matching theory has been extensively applied to a wide range of practical problems.

While matching theory has been applied to various practical problems, it has become apparent that the theory needs extension in some directions. First, many matching problems in real-life situations need to satisfy various constraints, and matching theory should be extended to accommodate those constraints. Second, many matching problems take place over time, and therefore, there is a need to extend matching theory to a dynamic context. The two papers in the second half of this special issue make important contributions to achieve those essential goals.

The paper by Kamada and Kojima offers a general framework to analyze matching problems with distributional constraints. A leading example is to avoid too much concentration of medical doctors in urban areas. A possible way to address this problem is to impose some constraints on the maximum number of doctors in each geographical region, and in fact, the Japanese government introduced such constraints to the original design of the matching mechanism. A companion paper by the same authors (Kamada and Kojima 2015) proposed an alternative mechanism that has some desirable properties. The proposed mechanism, however, is tailored to the specific problem, and therefore, it is not clear how to generalize it to accommodate a wider range of constraints. The present paper by Kamada and Kojima (2015) formulates policy goals of regions and proposes a general mechanism that respects the regional policy goals. The paper goes on to show that their model subsumes the mechanism proposed in their companion paper and that it improves upon the mechanism currently used in Japan.

The last paper in this special issue by Kurino carefully analyzes some fundamental issues in dynamic matching. It concerns one-to-one matching where the same set of agents are repeatedly matched over time. Moreover, agents are assumed to have time-additive utility. Even in this simplest possible dynamic environment, the paper shows, by means of a series of illuminating examples, extending the notion of stability to a dynamic context is by no means straightforward. One of the issues, for example, is whether a given coalition of agents, who propose an alternative dynamic matching, has an incentive to follow such an alternative. The paper proposes the "right" stability notion, and it is shown that a history-independent repetition of a 
side-optimal matching satisfies the proposed stability condition. This result is not only theoretically important, but it is also significant in practical applications, given the wide-spread use of the side-optimal mechanism (the deferred acceptance algorithm).

Acknowledgements As the guest editors of this special issue, we would like to thank the referees for providing their reports in a timely manner and the JER editorial office for the effective handling of the papers. We are also grateful for financial support from the grants acknowledged by the authors in this special issue.

\section{References}

Camerer, C. F., Ho, T. H., \& Chong, J. K. (2004). A cognitive hierarchy model of games. The Quarterly Journal of Economics, 119, 861-898.

Gale, D., \& Shapley, L. S. (1962). College admissions and the stability of marriage. American Mathematical Monthly, 69, 9-15.

Harsanyi, J. C. (1967-1968). Games with incomplete information played by Bayesian players, I-III. Management Science, 14, 159-183 (Part I), 320-334 (Part II), 486-502 (Part III).

Imai, H., \& Okada, A. (2005). Symposium on game theory and economics: Introduction. Japanese Economic Review, 56, 249-250.

Kajii, A., \& Morris, S. (1997). The robustness of equilibria to incomplete information. Econometrica, 65, 1283-1309.

Kamada, Y., \& Kojima, F. (2015). Efficient matching under distributional constraints: Theory and applications. American Economic Review, 105, 67-99.

Lucas, R. (1972). Expectations and the neutrality of money. Journal of Economic Theory, 4, 103-124.

Nagel, R. (1995). Unraveling in guessing games: An experimental study. American Economic Review, 85, $1313-1326$.

Okada, A. (1981). On the stability of perfect equilibrium points. International Journal of Game Theory, $10,67-73$.

Radner, R. (1962). Team decision problems. Annals of Mathematical Statististics, 33, 857-881.

Rubinstein, A. (1989). The electronic mail game: Strategic behavior under "almost common knowledge". American Economic Review, 79, 385-391.

Stahl, D. O., \& Wilson, P. W. (1994). Experimental evidence on players' models of other players. Journal of Economic Behavior and Organization, 25, 309-327.

Ui, T. (2001). Robust equilibria of potential games. Econometrica, 69, 1373-1380.

Publisher's Note Springer Nature remains neutral with regard to jurisdictional claims in published maps and institutional affiliations. 